Electronic Supplementary Information

\title{
Interplay of acid-base ratio and recycling on the pretreatment performance of the protic ionic liquid monoethanolammonium acetate
}

Pedro Y.S. Nakasu ${ }^{t \neq}$, Coby J. Clarke ${ }^{\ddagger}$, Sarita C. Rabelo", Aline C. Costa ${ }^{\dagger}$, Agnieszka Brandt-

Talbot ${ }^{\star}$ Jason P. Hallett ${ }^{*}$

${ }^{\dagger}$ Process and Products Development Department, Faculty of Chemical Engineering, State

University of Campinas (UNICAMP), Av. Albert Einstein, 500, Campinas/SP-Brazil

Department of Chemical Engineering, Imperial College London, South Kensington, London-

UK

" Department of Bioprocess and Biotechnology, College of Agricultural Sciences, São Paulo

State University (UNESP), Av. Universitária, 3780, Botucatu/SP-Brazil

*Corresponding author e-mail: j.hallett@imperial.ac.uk

Number of pages: 23

Number of figures: 21

Number of tables: 5 


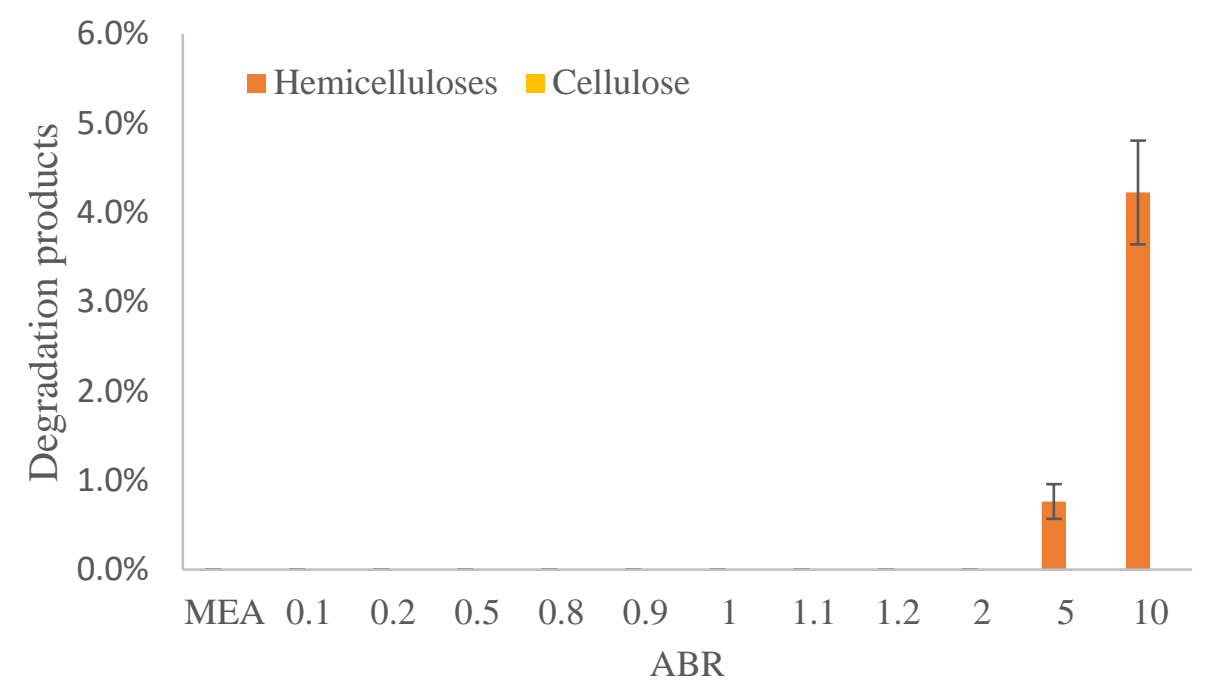

Figure S1. Degradation products in the liquid fractions obtained in the ABR experiments expressed as percentage of hemicelluloses (orange) - in the form of furfural - and cellulose (yellow) - in the form of 2-hydroxymethylfurfural (HMF), formic and levulinic acids.



Figure S2. Hydrolysis residues (purple bars) in oven dry basis obtained after 72 h of enzymatic saccharification and estimated hydrolysis residues (orange line) based on glucan and hemicellulose conversions in saccharification. 


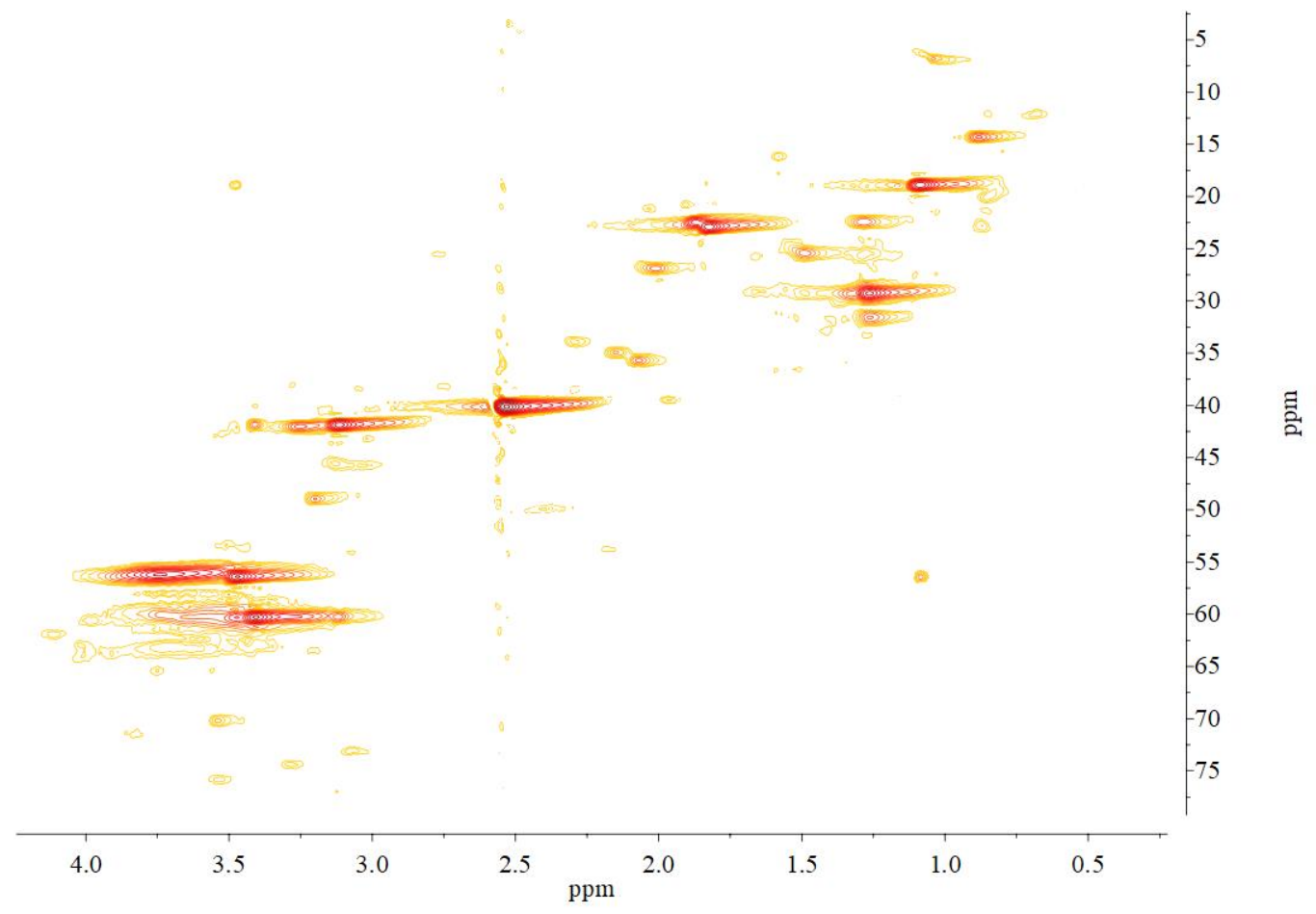

Figure S3. Aliphatic region of the HSQC spectrum of [MEA][OAc] lignin. Pretreatment conditions with [MEA][OAc] were $150^{\circ} \mathrm{C}, 30 \%$ water content, $15 \%$ solids loading and $2 \mathrm{~h}$ of reaction time. 


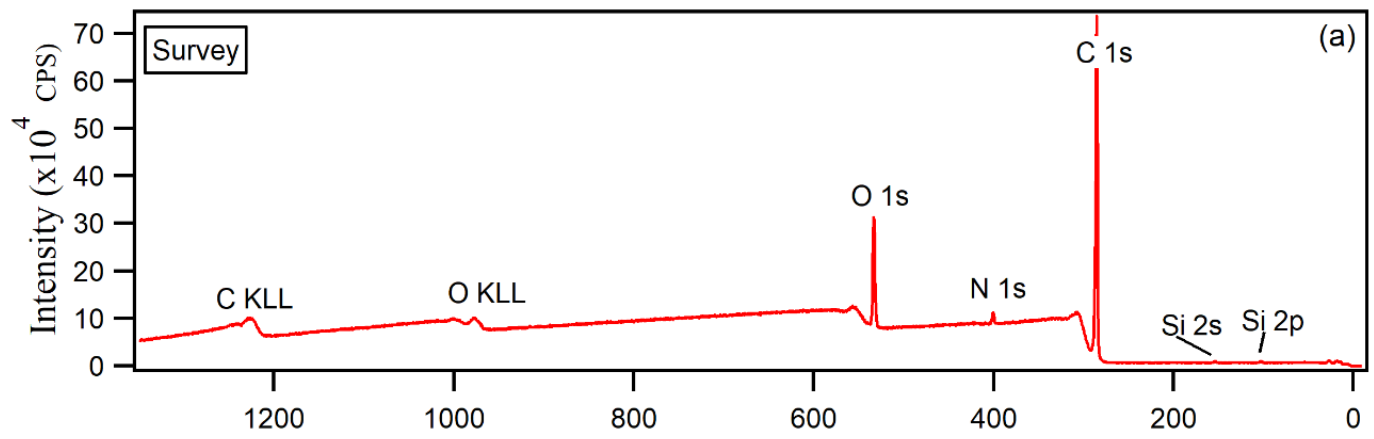

B.E. $(\mathrm{eV})$
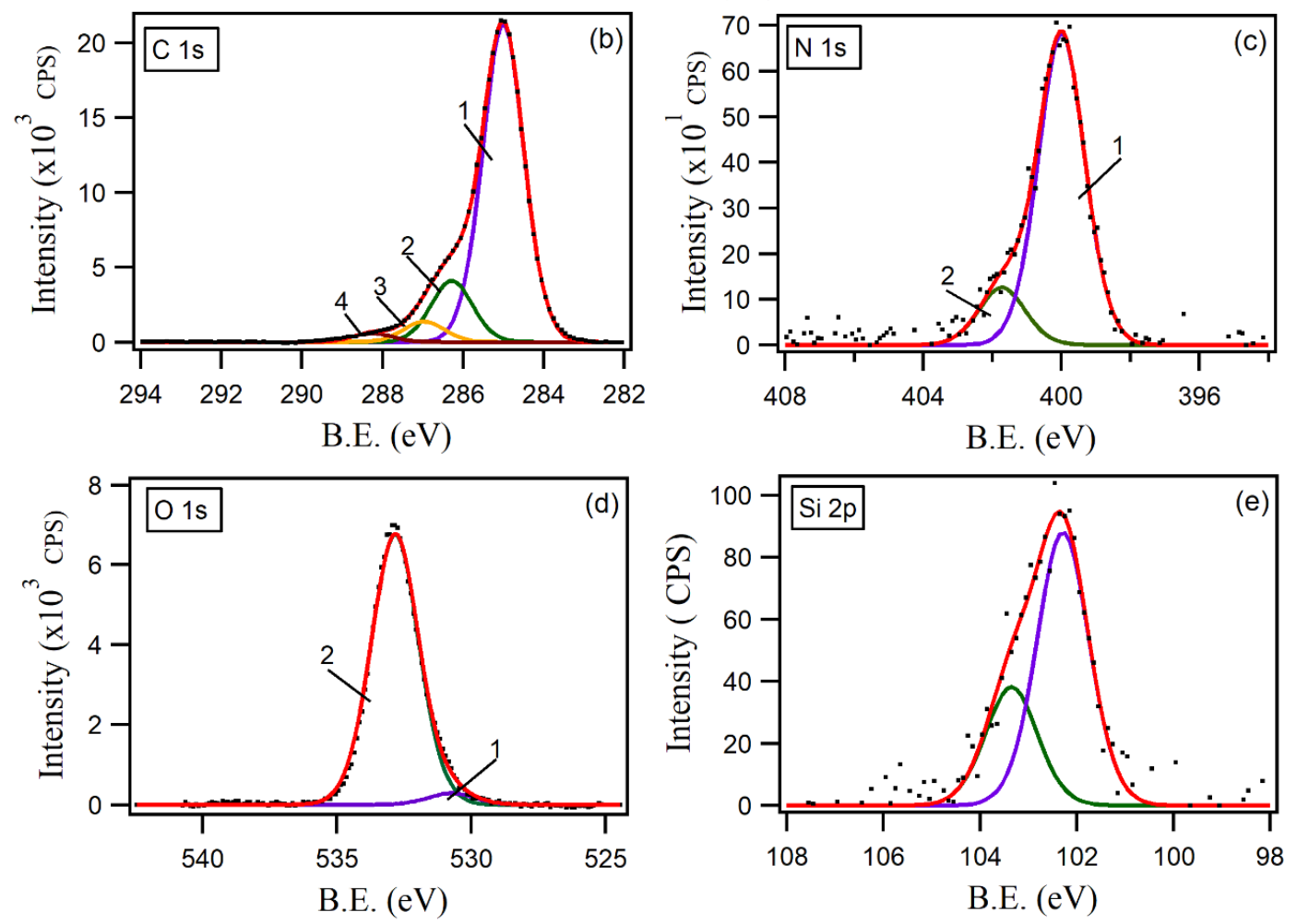

Figure S4. [MEA][OAc] 1 ABR lignin survey (a) and high resolution (b-f) photoemission spectra. 

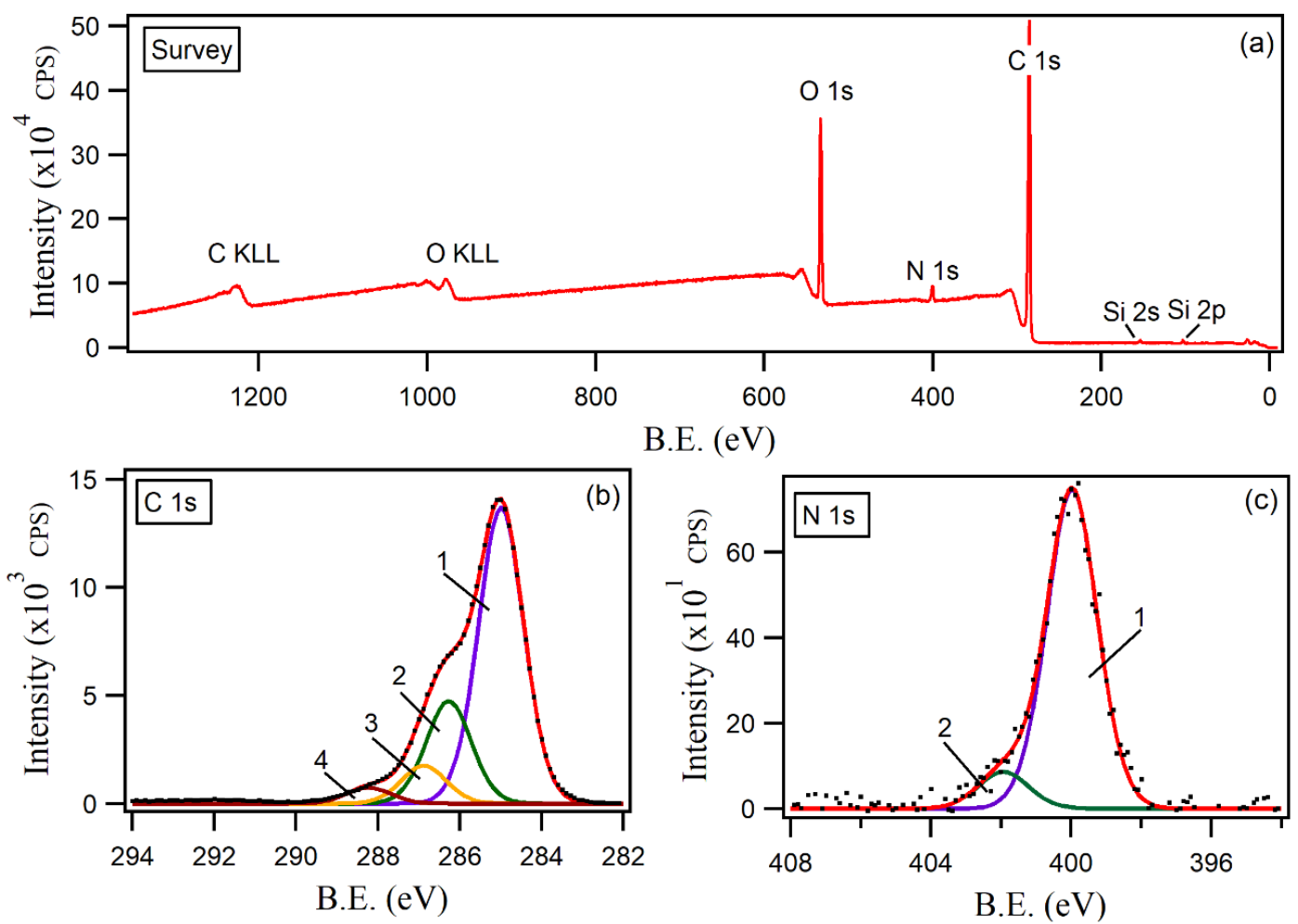

B.E. $(\mathrm{eV})$


Figure S5. [MEA][OAc] 0.8 ABR lignin survey $(a)$ and high resolution $(b-f)$ photoemission spectra. 


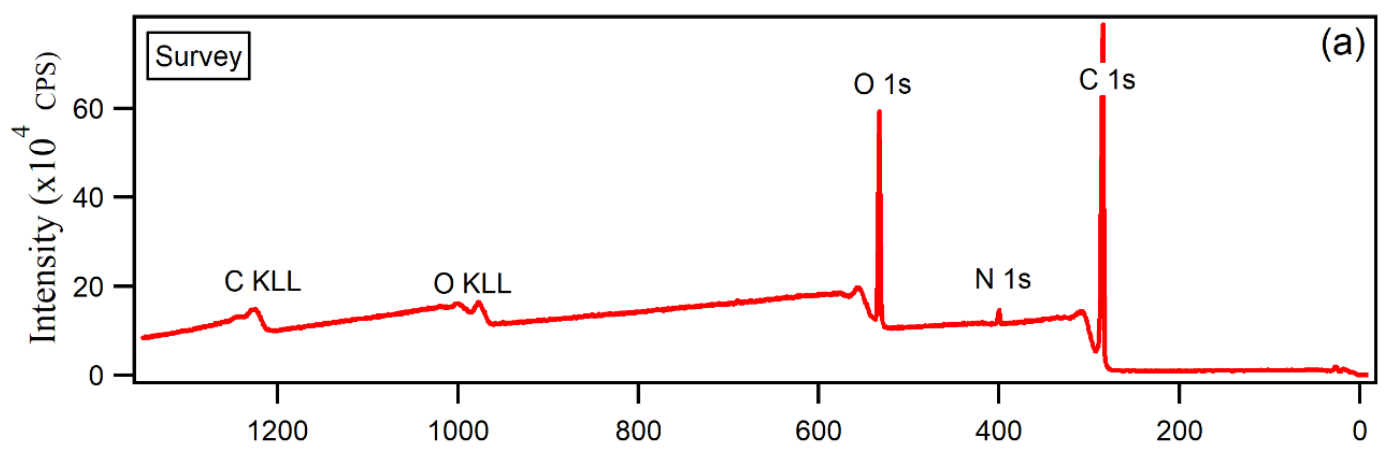

B.E. $(\mathrm{eV})$
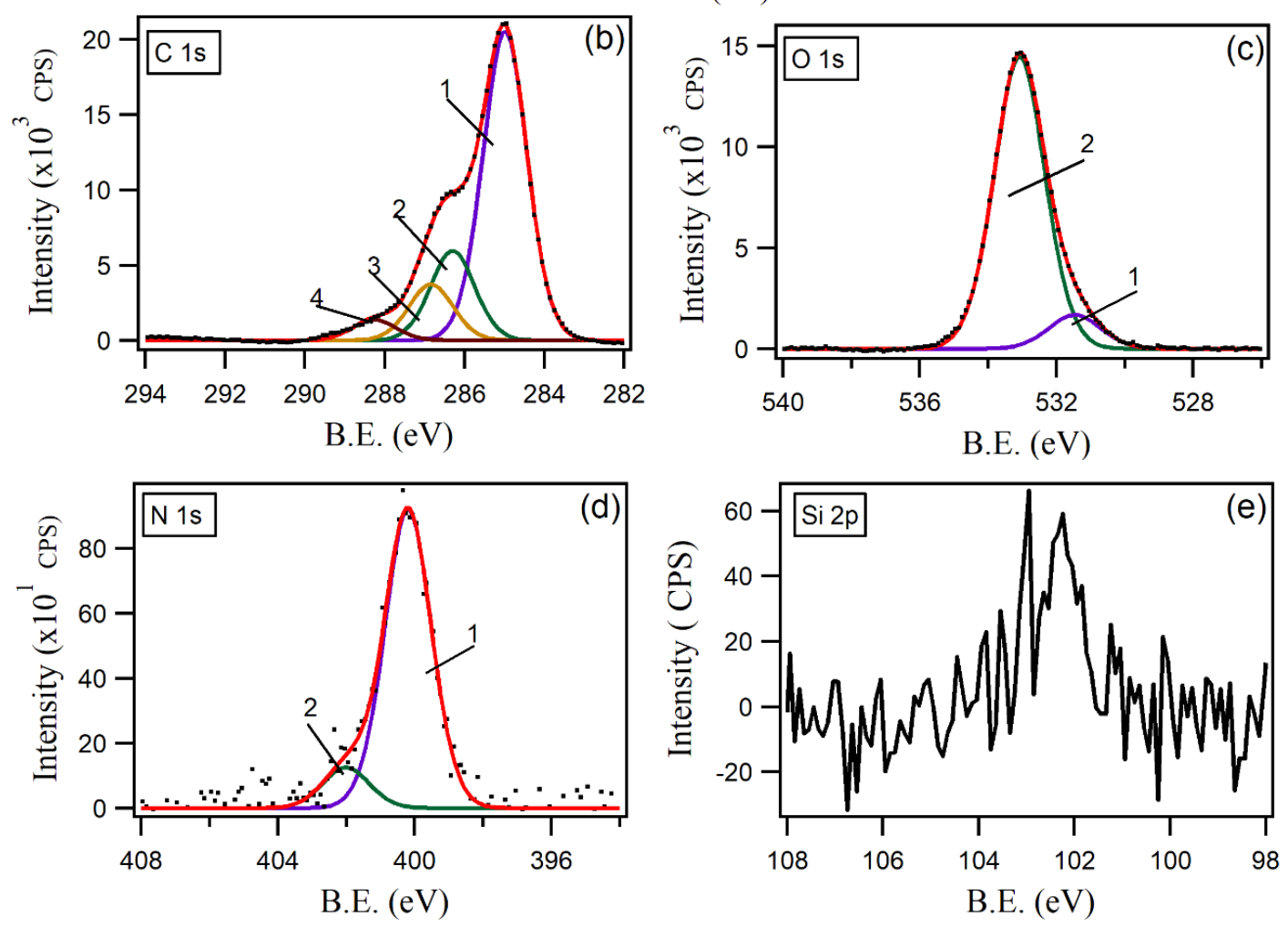

Figure S6. [MEA][OAc]2 ABR lignin survey (a) and high resolution (b-f) photoemission spectra. 


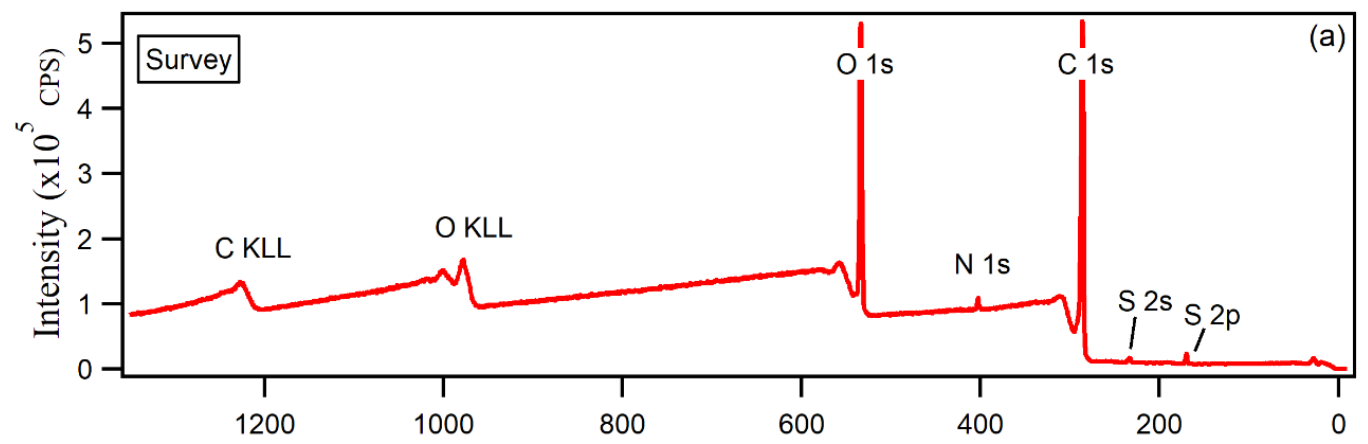

B.E. $(\mathrm{eV})$
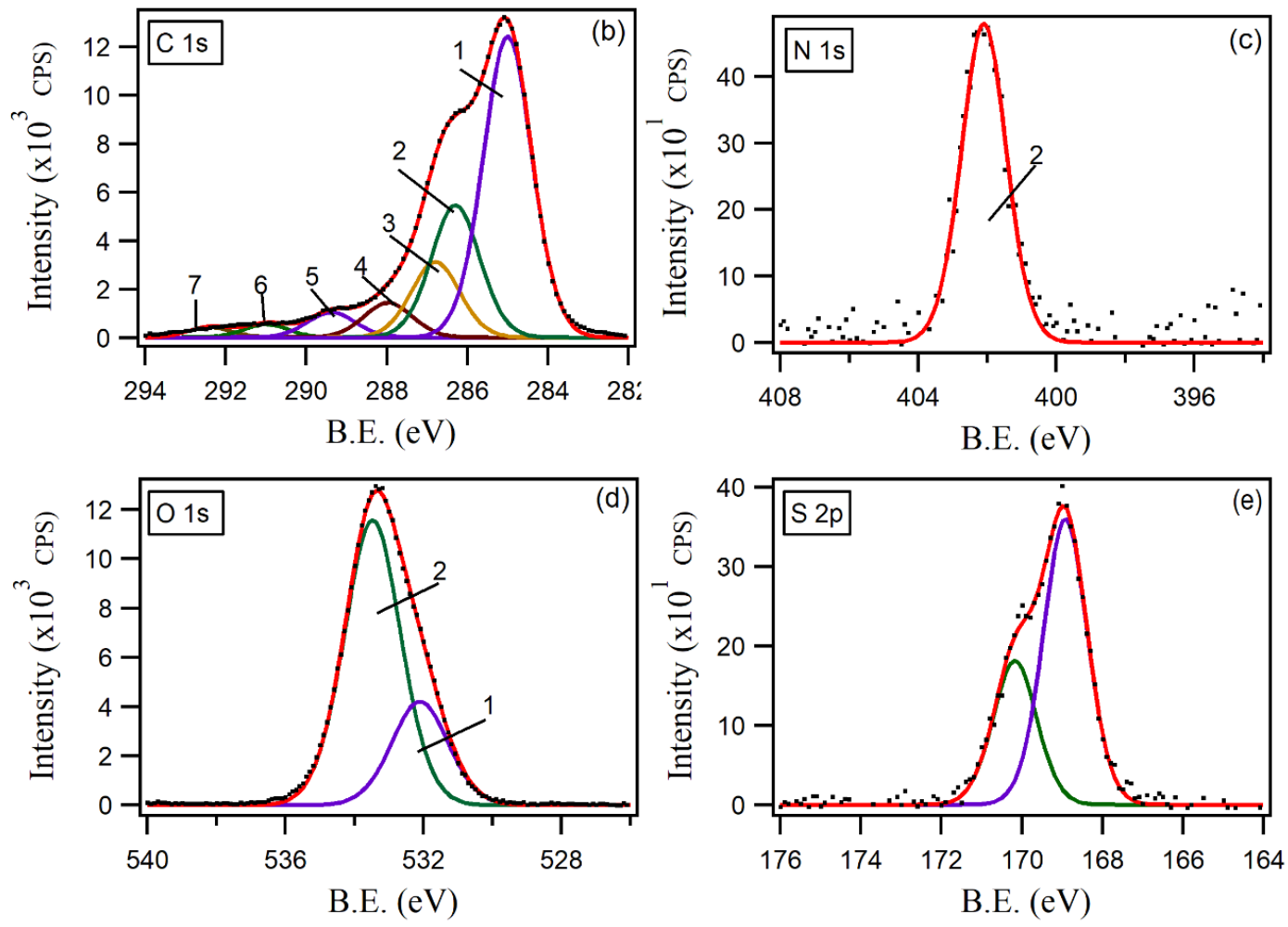

Figure S7. [TEA][HSO4] lignin survey (a) and high resolution (b-f) photoemission spectra. 


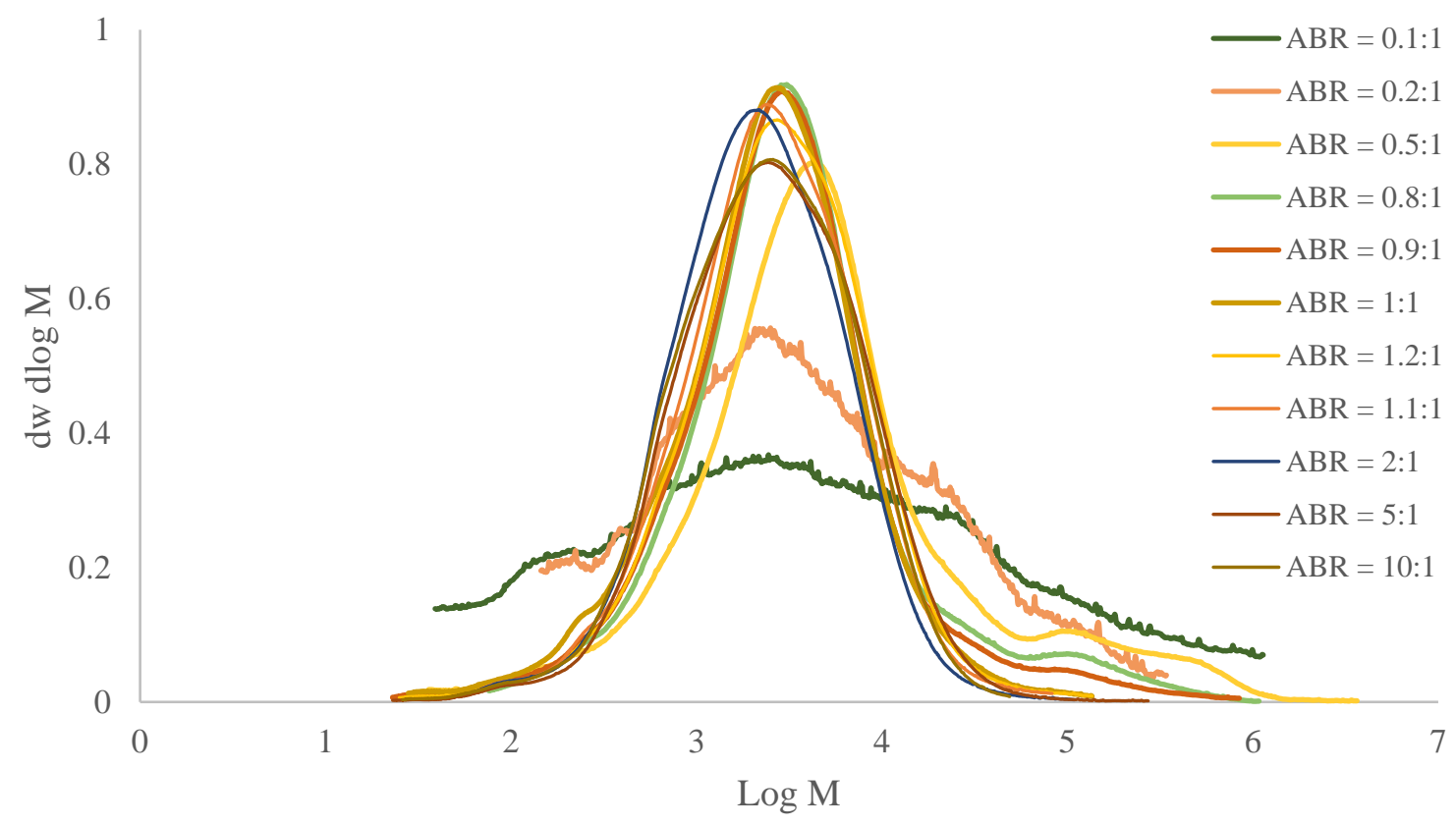

Figure S8. GPC profiles of the recovered lignins from the ABR experiments

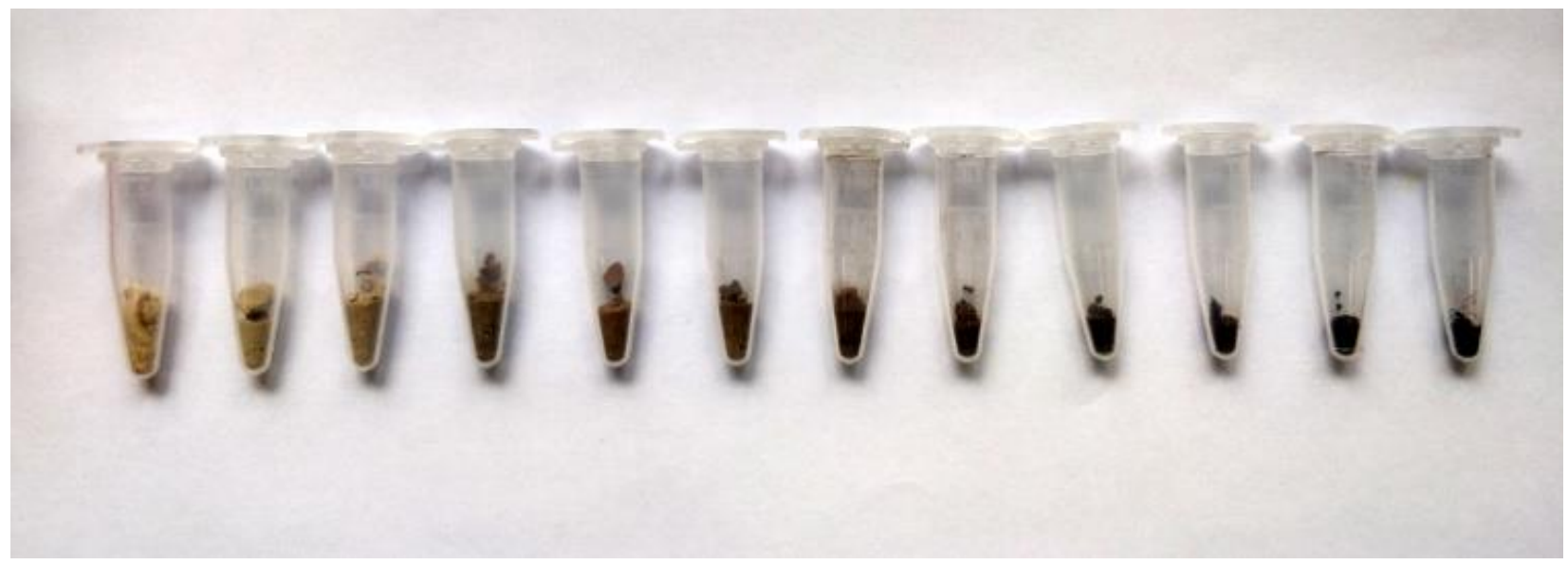

Figure S9. From left to right: lignins obtained from MEA, 0.1, 0.2, 0.5, 0.8, 0.9, 1.0, 1.1, 1.2, 2,5 and 10 ABR pretreatments. 
(a)

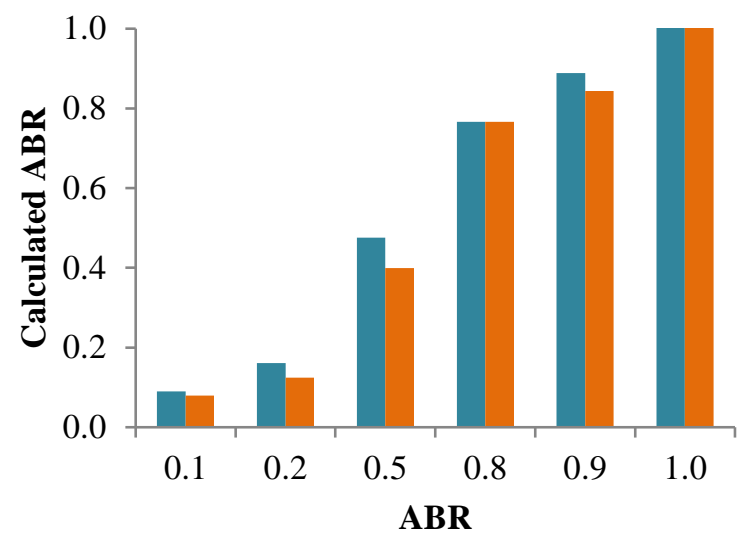

(b)

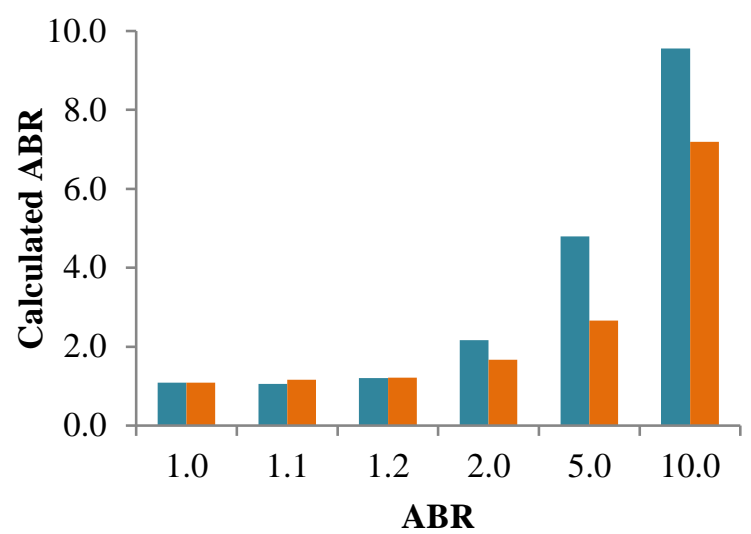

Figure S10. Calculated ABRs before ( ) and after ( ) pretreatment with [MEA][OAc] with excess base (a) and excess acid (b). Calculated ABRs were detemined by ${ }^{1} H$-NMR spectroscopy by integrating the peaks for the methyl group in the acetate anion and the more shielded methylene $(\delta=2.76)$ group in the MEA cation.

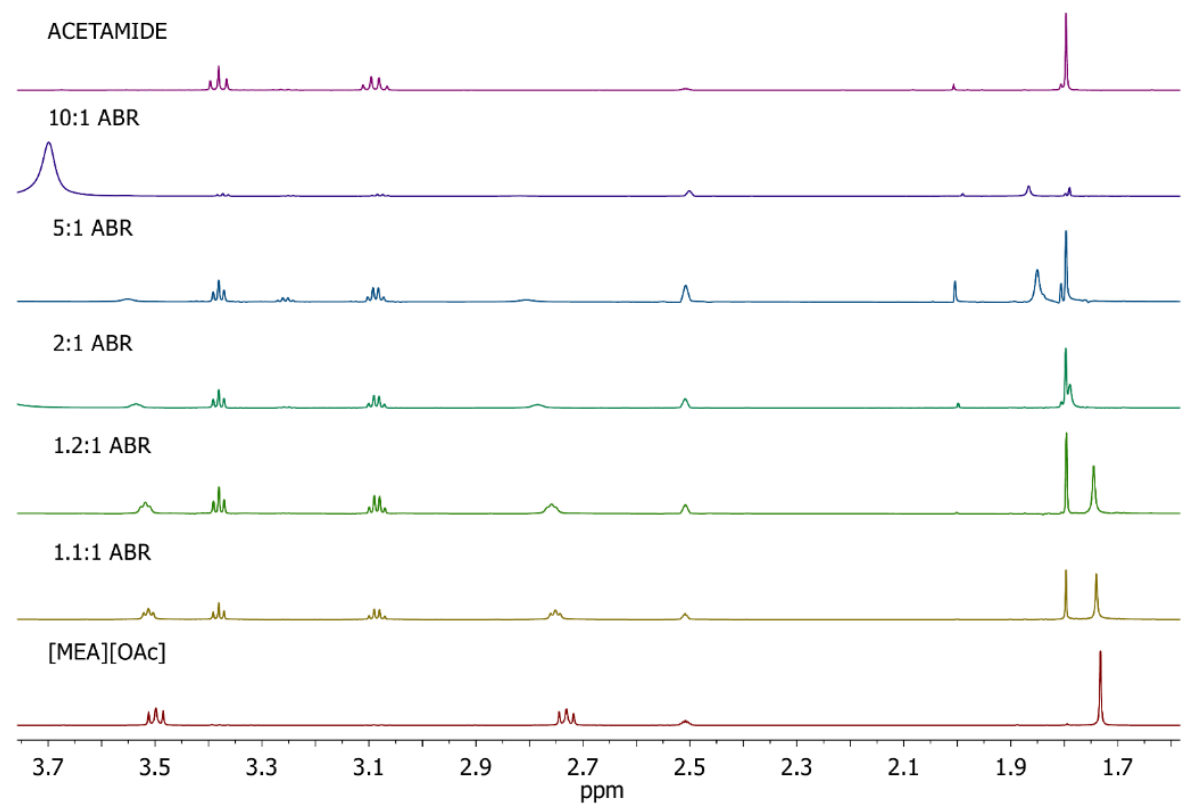

Figure S11. ${ }^{1} H$-NMR spectra of acid-base mixtures with ABR from 1.1:1 to 10:1. Acetamide and [MEA][OAc] spectra were also shown as a matter of comparison. 


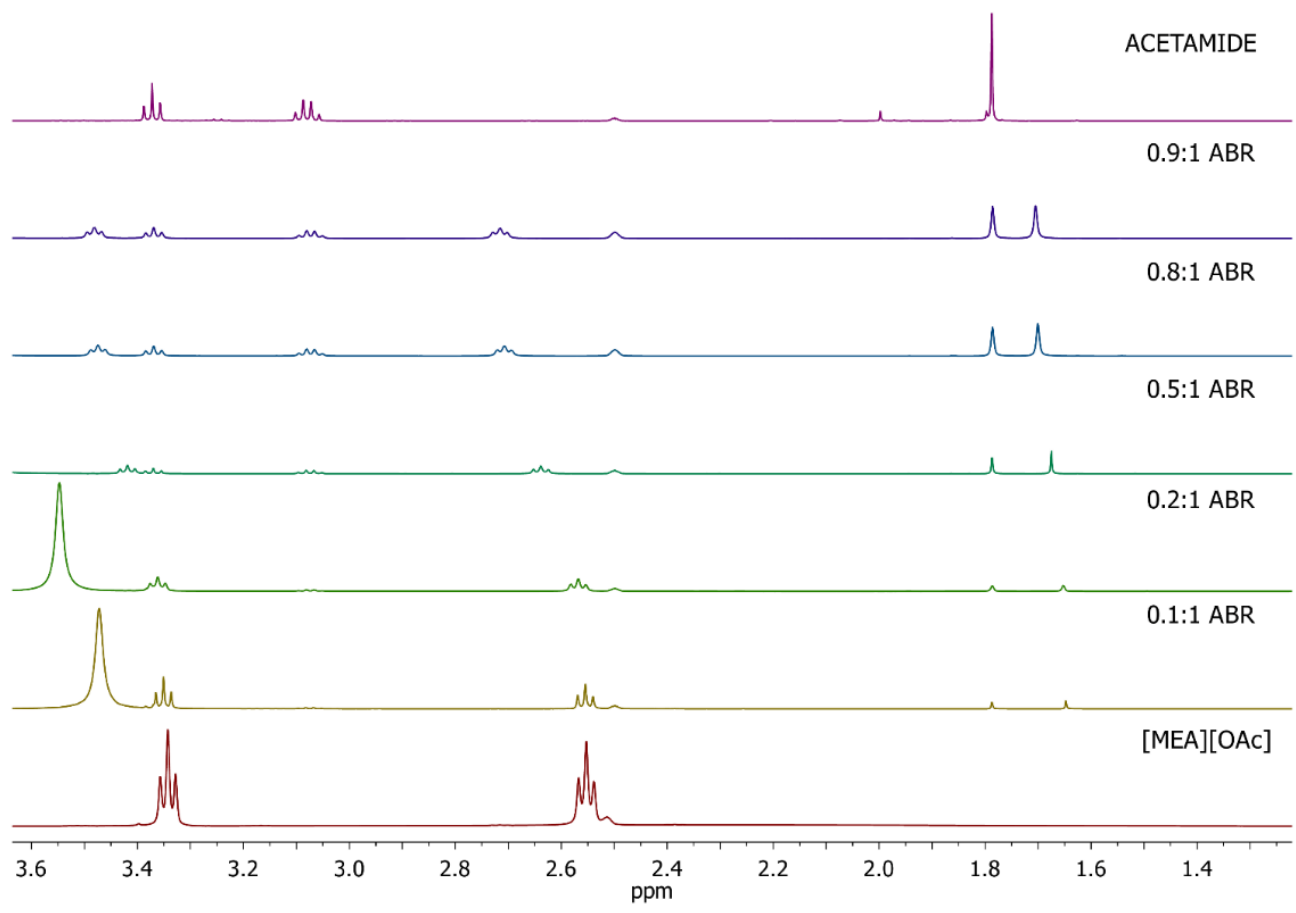

Figure S12. ${ }^{1} \mathrm{H}$-NMR spectra of acid-base mixtures with ABR from 0.1 to 0.9. Acetamide and [MEA][OAc] spectra were also shown as a matter of comparison.

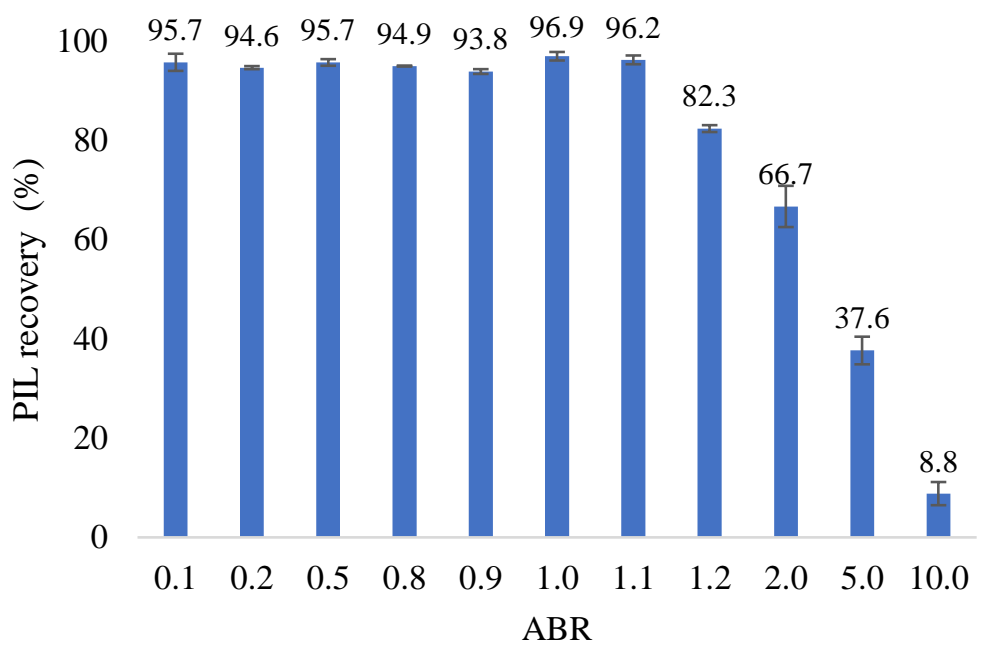

Figure S13. PIL recovery rates for the ABR experiments. The error bars were calculated based on duplicates. 


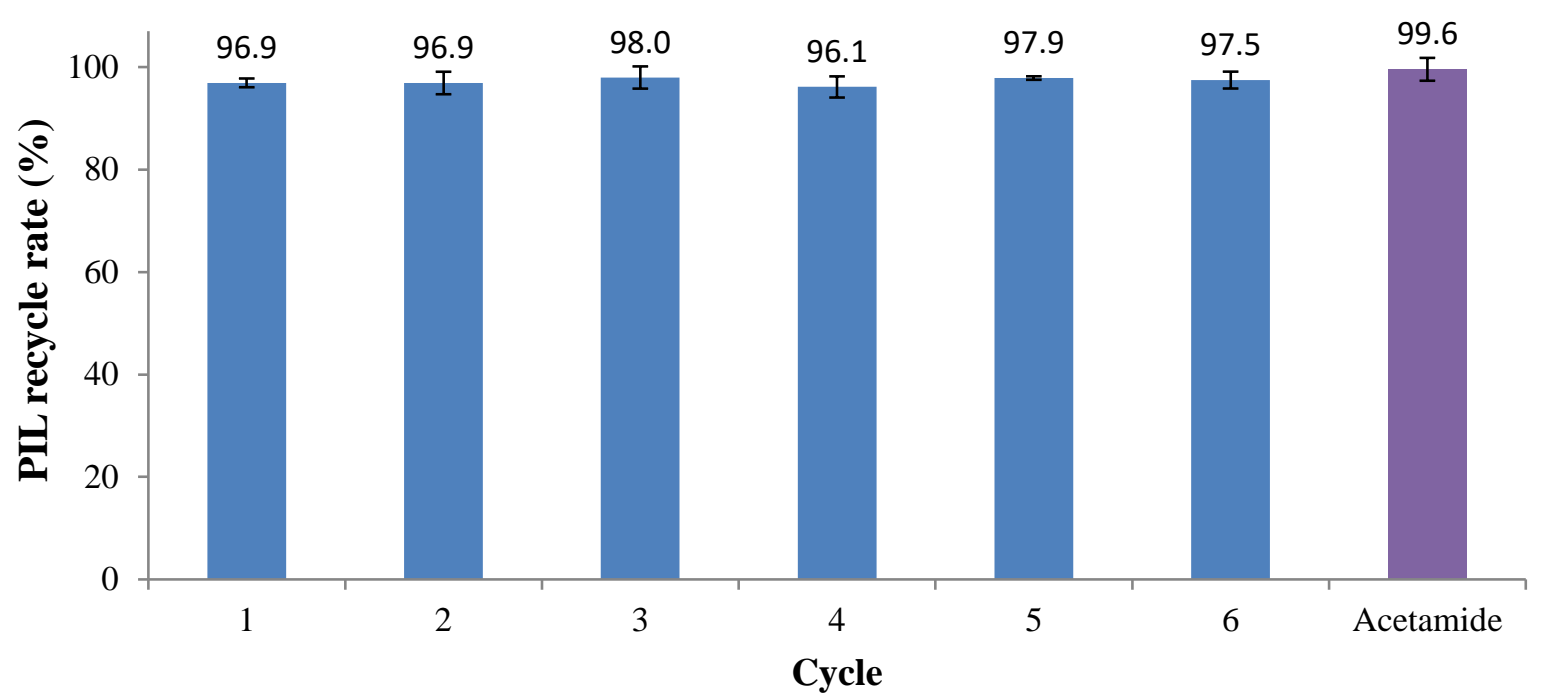

Figure S14. Recovery rates obtained for [MEA][OAc] recycle with 1 ABR. The error bars were calculated based on triplicates of the experiment. A pretreatment control with pure acetamide (purple bar) was shown as a matter of comparison. Pretreatment conditions with [MEA][OAc] were $150^{\circ} \mathrm{C}, 30 \%$ water content, $15 \%$ solids loading and $2 \mathrm{~h}$ of reaction time.

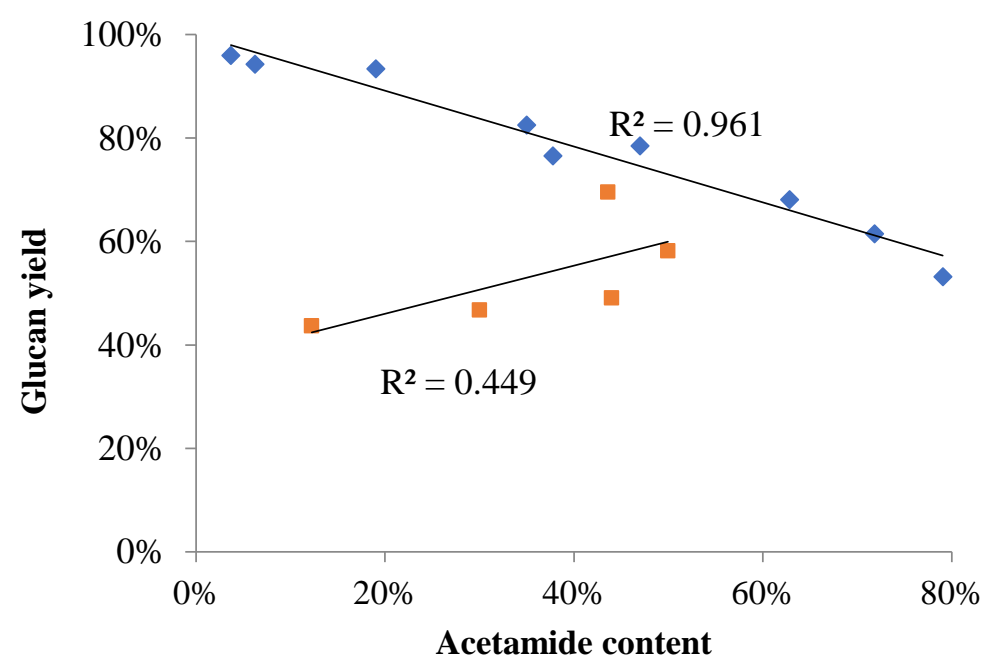

Figure S15. Correlation between glucose yield after $72 \mathrm{~h}$ of enzymatic saccharification and acetamide content in the recovered IL. Blue data points: low ABRs and recycle of [MEA][OAc] with 1.0 ABR. Orange data points: high ABR ratios. 


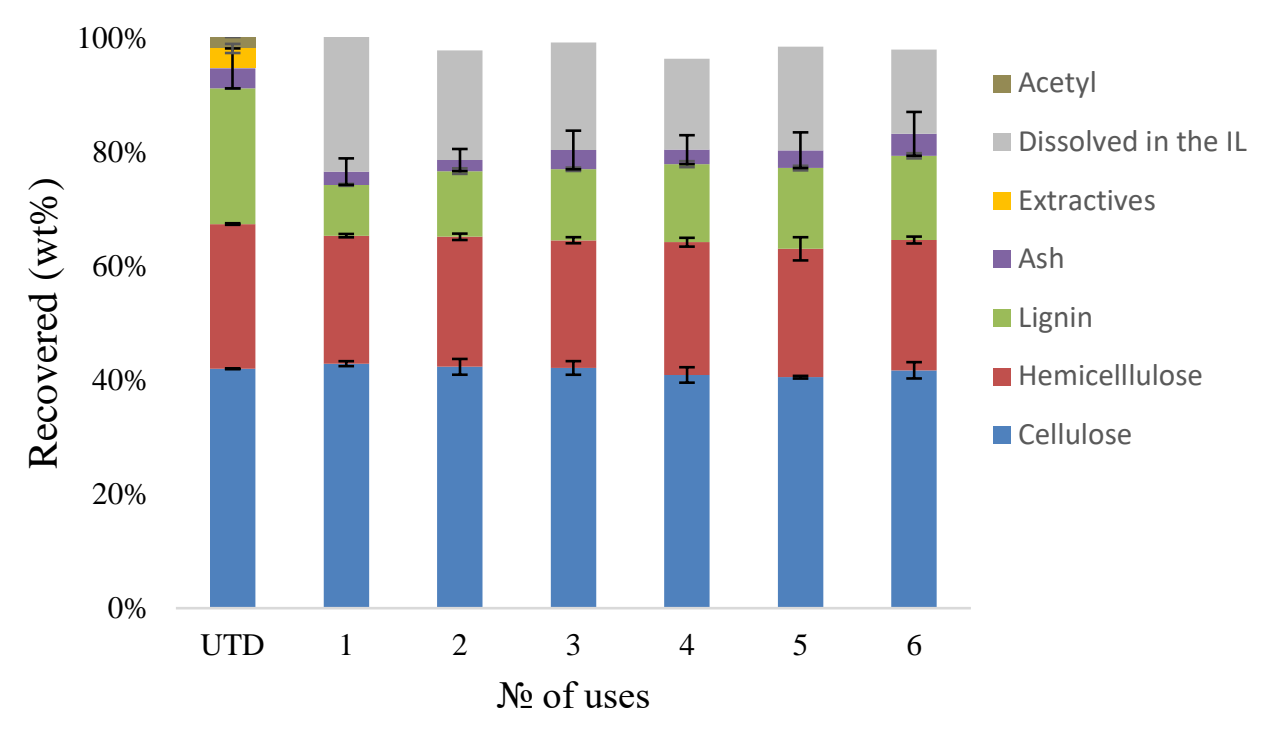

Figure S16. Pulp yields (non-grey fill) and pulps composition on the pretreatment of bagasse with recycling of [MEA] [OAc] with different $1.0 \mathrm{ABR}, 20 \mathrm{wt} \%$ water at $150^{\circ} \mathrm{C}$ for $2 \mathrm{~h}$ and $15 \mathrm{wt} \%$ solids loading. Untreated (UTD) sugarcane bagasse was also displayed.

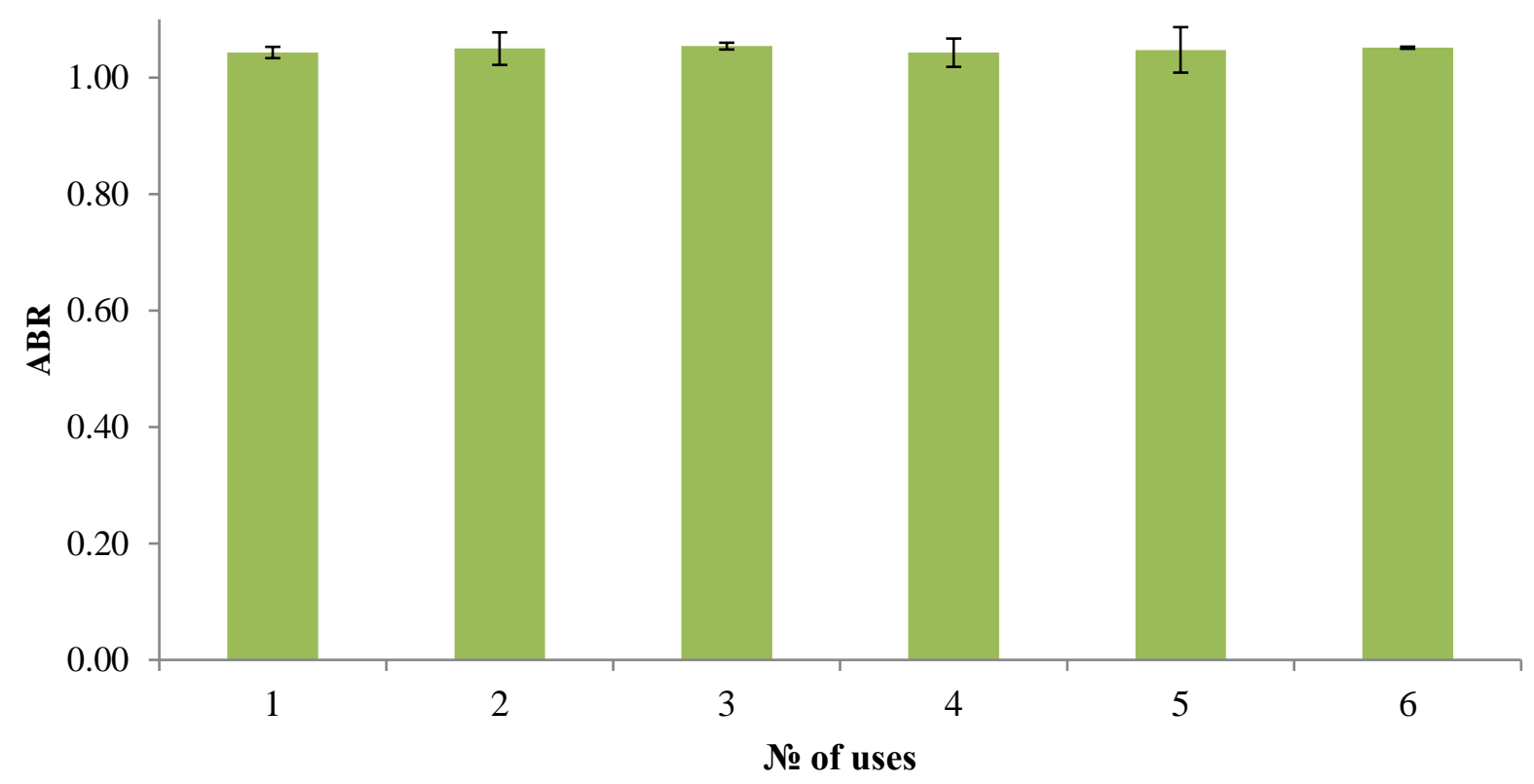

Figure S17.. ABRs (green bars) after repeated use of [MEA][OAc] with 1.0 ABR for pretreatment of sugarcane bagasse. 


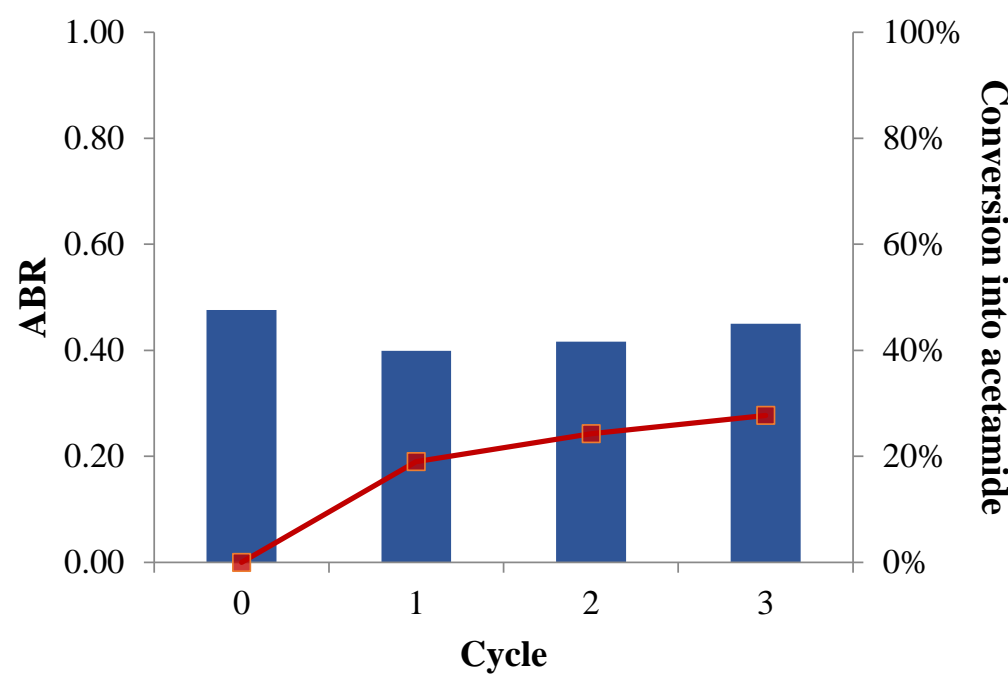

Figure S18. ABRs of the solution after pretreatment of bagasse with [MEA][OAc] with 0.5:1 ABR during recycling. Acetamide content indicated by the red data points (- - ).

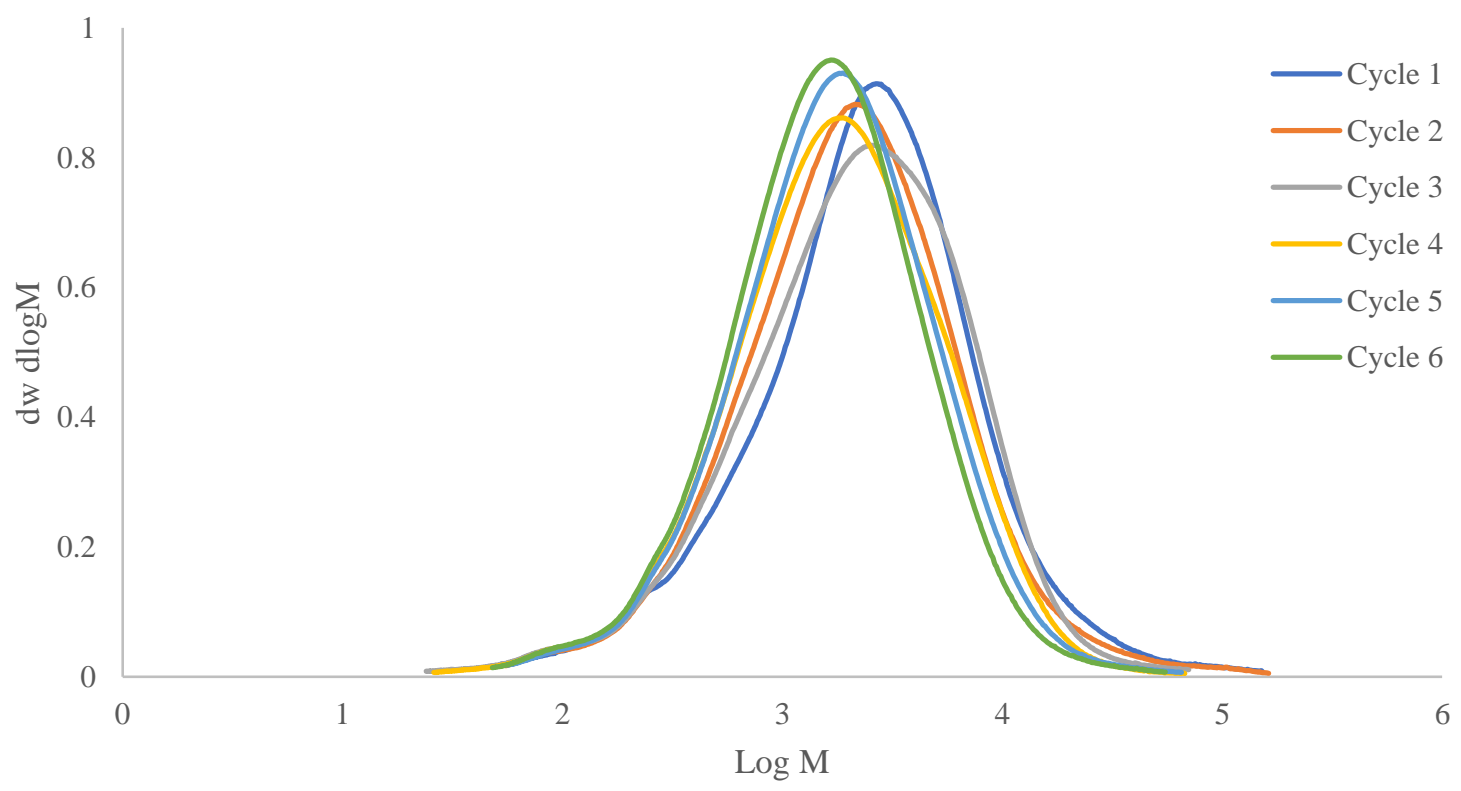

Figure S19. GPC profiles of the recovered lignins from the recycle experiments with 1 ABR. 


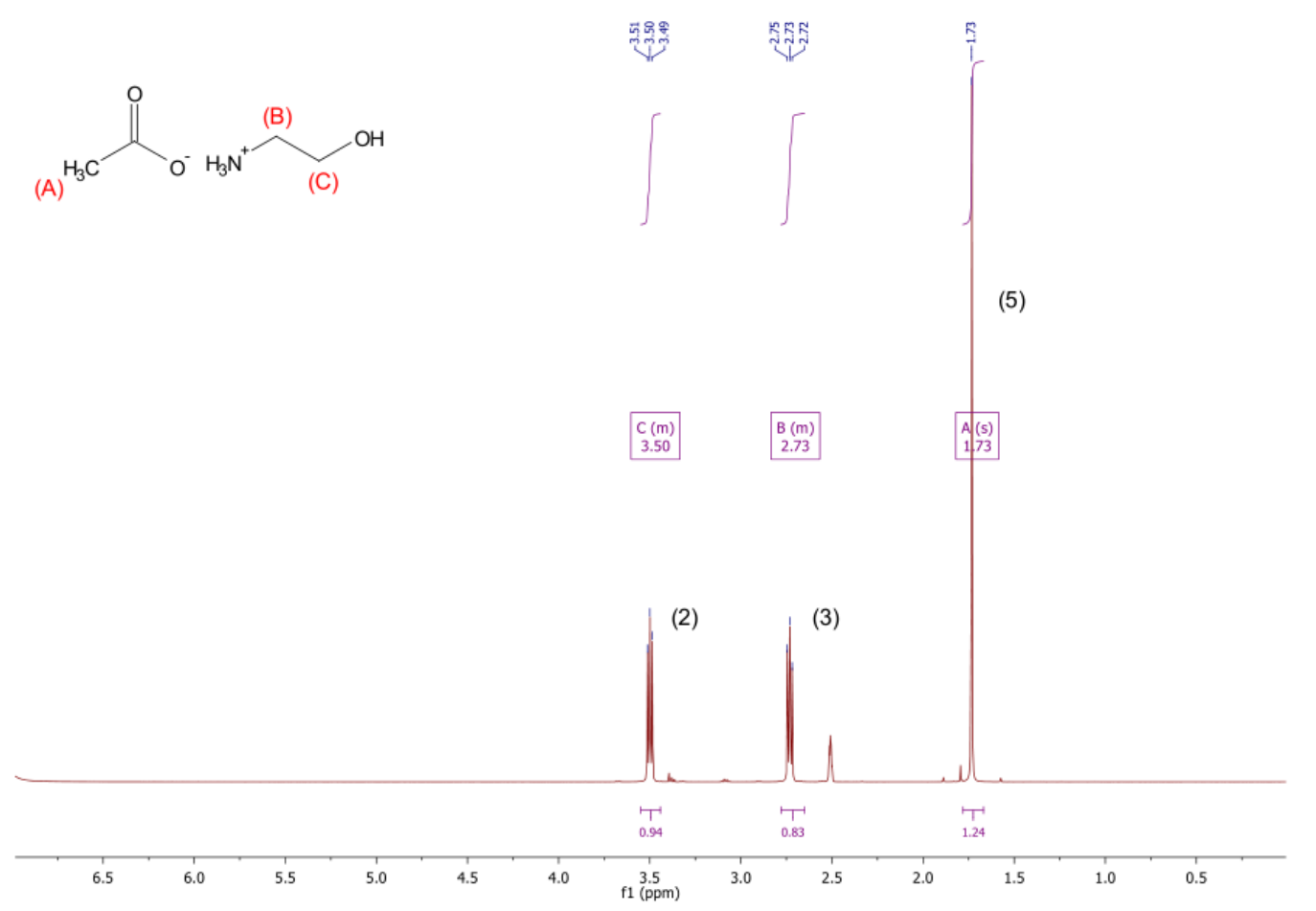

Figure S20. NMR-H $H^{1}$ spectrum of monoethanolammonium acetate in DMSO-d .
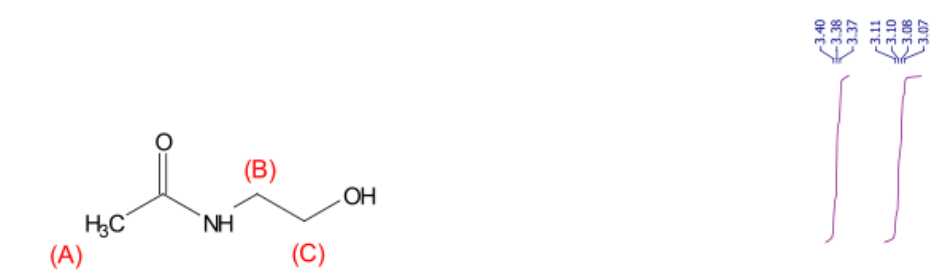

(C) 
Table S1. Elemental analysis of lignins obtained in the ABR experiments.

\begin{tabular}{l|rr|rr|rr}
\hline Sample & $\mathbf{C}(\boldsymbol{\%})$ & \multicolumn{1}{c|}{ SD (\%) } & H (\%) & SD (\%) & N (\%) & \multicolumn{1}{c}{ SD (\%) } \\
\hline MEA & 15.90 & 0.029 & 3.59 & 0.029 & 3.08 & 0.050 \\
$\mathbf{0 . 1}$ & 21.80 & 0.330 & 4.00 & 0.330 & 2.79 & 0.050 \\
$\mathbf{0 . 2}$ & 25.28 & 0.133 & 4.29 & 0.133 & 3.15 & 0.029 \\
$\mathbf{0 . 5}$ & 50.95 & 0.109 & 6.14 & 0.109 & 3.04 & 0.045 \\
$\mathbf{0 . 8}$ & 54.37 & 0.037 & 6.41 & 0.037 & 3.63 & 0.023 \\
$\mathbf{0 . 9}$ & 57.51 & 0.015 & 6.62 & 0.015 & 2.83 & 0.021 \\
$\mathbf{1 . 0}$ & 58.56 & 0.151 & 6.56 & 0.151 & 3.63 & 0.053 \\
$\mathbf{1 . 1}$ & 60.74 & 0.036 & 6.43 & 0.036 & 2.33 & 0.032 \\
$\mathbf{1 . 2}$ & 61.20 & 0.092 & 6.43 & 0.092 & 2.37 & 0.124 \\
$\mathbf{2 . 0}$ & 62.10 & 0.017 & 6.24 & 0.017 & 2.59 & 0.083 \\
$\mathbf{5 . 0}$ & 62.35 & 0.140 & 6.07 & 0.140 & 2.86 & 0.071 \\
$\mathbf{1 0}$ & 62.64 & 0.037 & 5.93 & 0.037 & 2.74 & 0.046 \\
\hline
\end{tabular}


Table S2. Binding energies (eV) for the photoemissions of each lignin sample. All high-resolution XP spectra are charge corrected to the aliphatic component $1(285.0 \mathrm{eV})$ and the most intense signals (i.e. $2 p$ 3/2) are reported for doubly degenerate photoemissions.

\begin{tabular}{|c|c|c|c|c|c|c|c|c|c|c|c|}
\hline \multirow{3}{*}{ Component } & \multicolumn{11}{|c|}{ Binding Energy $(\mathrm{eV})$} \\
\hline & \multicolumn{4}{|l|}{$\mathrm{C} 1 \mathrm{~s}$} & \multicolumn{2}{|l|}{$\mathrm{N} 1 \mathrm{~s}$} & \multirow{2}{*}{$\begin{array}{l}\mathrm{O} 1 \mathrm{~s} \\
1\end{array}$} & \multicolumn{2}{|c|}{ Si $2 p$} & $S 2 p$ & $\mathrm{Ca} 2 \mathrm{p}$ \\
\hline & 1 & 2 & 3 & 4 & $1^{\mathrm{a}}$ & $2^{\mathrm{a}}$ & & 2 & 1 & 2 & \\
\hline MEA & 285.0 & 286.2 & 287.1 & 288.5 & 400.2 & 402.2 & 531.2 & 533.0 & 102.6 & 103.5 & 347.6 \\
\hline $1: 1$ & 285.0 & 286.3 & 287.0 & 288.3 & 400.0 & 401.7 & 530.7 & 532.8 & 102.4 & & \\
\hline $1: 0.8$ & 285.0 & 286.3 & 286.9 & 288.2 & 400.0 & 401.9 & 531.2 & 533.0 & 102.5 & & \\
\hline $2: 1$ & 285.0 & 286.3 & 286.8 & 288.3 & 400.2 & 402 & 531.5 & 533.1 & & & \\
\hline$[\mathrm{TEA}]\left[\mathrm{HSO}_{4}\right]$ & 285.0 & 286.3 & 286.8 & 288.1 & & 402.1 & & 533.2 & & 168.9 & \\
\hline
\end{tabular}

${ }^{a}$ Components identified as neutral (1) and cationic (2) nitrogen 
Table S3. Molar weight of precipitate obtained for at different ABRs. Sugarcane bagasse was pretreated with [MEA][OAc] and a precipitate generated by removing excess wash water. Mn is the average molecular weight, $M w$ the number average weight and $P D$ for polidispersity. $S D=$ standard deviation .

\begin{tabular}{rcc|cc|cc}
\hline \multicolumn{1}{c}{ ABR } & $\mathbf{M}_{\mathbf{n}}$ & SD & $\mathbf{M}_{\mathbf{w}}$ & $\mathbf{S D}$ & $\mathbf{P D}$ & $\mathbf{S D}$ \\
\hline $\mathbf{0 . 1}$ & 1530 & 47.6 & 29310 & 487.2 & 19.2 & 0.28 \\
$\mathbf{0 . 2}$ & 1384 & 248.2 & 26853 & 1536.7 & 18.4 & 0.65 \\
$\mathbf{0 . 5}$ & 1492 & 94.8 & 27043 & 1268.6 & 18.1 & 0.30 \\
$\mathbf{0 . 8}$ & 1460 & 90.5 & 16275 & 420.1 & 11.2 & 0.40 \\
$\mathbf{0 . 9}$ & 1240 & 138.6 & 9288 & 1294.7 & 7.6 & 1.89 \\
$\mathbf{1}$ & 1072 & 75.0 & 4742 & 75.7 & 4.4 & 0.24 \\
$\mathbf{1 . 1}$ & 1330 & 73.5 & 4410 & 113.8 & 3.3 & 0.10 \\
$\mathbf{1 . 2}$ & 1215 & 5.7 & 5199 & 306.2 & 4.3 & 0.23 \\
$\mathbf{2}$ & 1194 & 36.1 & 3705 & 43.8 & 3.1 & 0.06 \\
$\mathbf{5}$ & 1300 & 65.8 & 4576 & 118.1 & 3.5 & 0.27 \\
$\mathbf{1 0}$ & 1165 & 31.1 & 3905 & 31.1 & 3.4 & 0.12 \\
\hline
\end{tabular}

Table S4. Elemental analysis of pulps obtained in the ABR experiments. Raw bagasse and monoethanolamine (MEA) pretreated samples are added for comparison.

\begin{tabular}{ccc|cc|cc}
\hline Sample & $\mathbf{C}(\boldsymbol{\%})$ & SD $(\%)$ & $\mathbf{H}(\boldsymbol{\%})$ & SD (\%) & $\mathbf{N}(\boldsymbol{\%})$ & SD (\%) \\
\hline $\begin{array}{c}\text { Raw } \\
\text { bagasse }\end{array}$ & 44.35 & 0.843 & 6.03 & 0.090 & 0.22 & 0.053 \\
MEA & 39.94 & 2.058 & 6.05 & 0.308 & 0.09 & 0.006 \\
$\mathbf{0 . 1}$ & 41.41 & 0.203 & 6.22 & 0.041 & 0.09 & 0.006 \\
$\mathbf{0 . 2}$ & 41.10 & 0.627 & 6.09 & 0.088 & 0.06 & 0.040 \\
$\mathbf{0 . 5}$ & 40.26 & 3.111 & 5.86 & 0.464 & 0.21 & 0.085 \\
$\mathbf{0 . 8}$ & 42.21 & 0.206 & 6.18 & 0.035 & 0.25 & 0.050 \\
$\mathbf{0 . 9}$ & 42.71 & 0.248 & 6.21 & 0.029 & 0.24 & 0.015 \\
$\mathbf{1 . 0}$ & 40.82 & 2.655 & 5.89 & 0.358 & 0.24 & 0.006 \\
$\mathbf{1}$ & 42.90 & 0.465 & 6.07 & 0.084 & 0.30 & 0.032 \\
$\mathbf{1 . 2}$ & 43.34 & 0.189 & 6.10 & 0.039 & 0.19 & 0.049 \\
$\mathbf{2}$ & 43.66 & 0.489 & 6.04 & 0.038 & 0.23 & 0.006 \\
$\mathbf{5}$ & 43.61 & 1.056 & 5.97 & 0.127 & 0.23 & 0.040 \\
$\mathbf{1}$ & 40.47 & 1.234 & 5.49 & 0.172 & 0.22 & 0.291 \\
\hline
\end{tabular}


Table S5. Molar weight parameters for the precipitate obtained in the recycling experiments with [MEA][OAc] with 1.0 ABR: Average molecular weight (Mn), number average weight $(\mathrm{Mw})$ and polidispersity $(P D)$. Standard deviations $(S D)$ were calculated based on triplicates of the samples.

\begin{tabular}{ccc|cc|cc}
\hline Cycle & Mn & SD & Mw & SD & PD & SD \\
\hline 1 & 1561.5 & 91.2 & 7161.5 & 400.9 & 4.6 & 0.0 \\
2 & 1397.0 & 69.3 & 5073.5 & 173.2 & 3.6 & 0.3 \\
3 & 1024.5 & 129.4 & 4065.5 & 638.5 & 4.0 & 1.1 \\
4 & 978.0 & 12.7 & 3233.0 & 1.4 & 3.3 & 0.0 \\
5 & 859.0 & 21.2 & 3141.5 & 126.6 & 3.7 & 0.2 \\
6 & 737.0 & 48.1 & 2718.0 & 56.6 & 3.7 & 0.3 \\
\hline
\end{tabular}

\section{Feedstock and pulp characterization}

\section{Moisture content}

The moisture content of raw bagasse, pretreated pulps, and hydrolysis residues was determined according to the NREL protocol 'Determination of Total Solids in Biomass and Total Dissolved Solids in Liquid Process Samples ${ }^{25}$ determination of total. The measurement was performed in triplicate for the raw bagasse and the hydrolysis residues and once per sample for the recovered pulps.

\section{Compositional analysis}

The composition of the raw bagasse and pretreated pulps was determined according to the NREL protocol 'Determination of Structural Carbohydrates and Lignin in Biomass'26. and 'Determination of Extractives in biomass'. ${ }^{27}$ In brief, the method consists of concentrated sulfuric acid hydrolysis followed by dilution with water and hydrolysis in an autoclave. The liquid was 
used to determine the carbohydrate content by HPLC. Gravimetric analysis of the remaining lignin-rich residue followed by calcination was used to determine the acid-insoluble lignin and ash content. Compositional analysis was performed in triplicate. Based on the lignin content in the pulps, the degree of delignification was calculated (Calculations section in the ESI).

\section{Lignin analysis}

\section{D-NMR - Heteronuclear single quantum coherence (HSQC)}

HSQC analysis was performed on the precipitated lignin. $20 \mathrm{mg}$ of lignin was dissolved in $0.25 \mathrm{~mL}$ of DMSO-d ${ }^{6}$ and the solution was transferred to a Shigemi tube. HSQC NMR spectra were recorded on a Bruker $600 \mathrm{MHz}$ spectrometer based on Gschwend et al. ${ }^{11}$

\section{Gel permeation chromatography (GPC)}

GPC analysis was performed using an Agilent 1260 Infinity instrument equipped with a Viscotek column set (AGuard, A6000M, and A3000M) and RID detector was used for detection. DMSO containing $\operatorname{LiBr}\left(1 \mathrm{~g} \cdot \mathrm{L}^{-1}\right)$ was used as eluent at a flow rate of $0.4 \mathrm{~mL} \cdot \mathrm{min}^{-1}$ at a column temperature of $60^{\circ} \mathrm{C}$. Samples and standards were prepared according to Brandt et al. ${ }^{28}$. Standard deviations for the lignins were $\mathrm{Mn} \pm 100, \mathrm{Mw} \pm 300$ and $\mathrm{PDI} \pm 0.3$ or less.

\section{Elemental analysis}

CHNS/O analysis of the recovered lignins and pulps was determined using an Elementar VarioMICRO Cube equipped with a combustion column containing tungsten (IV) oxide operating at $1150{ }^{\circ} \mathrm{C}$, a reduction column containing reduced copper wires at $850{ }^{\circ} \mathrm{C}$ and two adsorption columns operating at $850^{\circ} \mathrm{C}$ and from 40 to $210^{\circ} \mathrm{C}$, respectively. The instrument is also equipped 
with a TCD detector operating at $60{ }^{\circ} \mathrm{C}$. Helium was used as carrier and flushing gas and the combustion was carried out by pulse injection of oxygen. Samples $(\sim 2 \mathrm{mg})$ were weighed and sealed in aluminium boats prior to analysis. Each sample was measured at least three times and the oxygen content was determined by subtraction $(\% \mathrm{O}=100-\% \mathrm{C}-\% \mathrm{H}-\% \mathrm{~N}-\% \mathrm{~S}-\%$ ash $)$.

\section{X-ray photoelectron spectroscopy (XPS)}

X-ray photoelectron spectra were recorded on a Thermo Fisher K-Alpha Photoelectron Spectrometer equipped with a $180^{\circ}$ double focussing hemispherical analyser, 128-channel detector, and monochromated Al Ka microfocused x-ray source $(\mathrm{h} v=1486.6 \mathrm{eV})$ operated at 6 $\mathrm{mA}$ emission current and $12 \mathrm{kV}$ anode bias. Prior to XPS measurements, samples were degassed on a Schlenk line at $<1 \times 10^{-2}$ mbar for $24 \mathrm{~h}$ before mounting 5-10 $\mathrm{mg}$ on a welled copper plate. After degassing to $<3 \times 10^{-7}$ mbar in the sample transfer chamber, samples were introduced to the analysis chamber, which operates with a base pressure of $<2 \times 10^{-9} \mathrm{mbar}$. A spot size of $400 \mu \mathrm{m}$ and pass energies of $200 \mathrm{eV}$ (survey) and $20 \mathrm{eV}$ (high resolution) were used during analysis. Typically, 60 scans with a dwell time of $50 \mathrm{~ms}$ were used for HR scans; hence each HR scan equals $\approx 10 \mathrm{~m}$ of X-ray exposure. Samples were prevented from charging with a dual-beam flood source.

Survey scans were quantified in Avantage 5.951 using smart backgrounds and ALTHERMO1 RSFs. High resolution scans were converted to .VAMAS files and processed in CasaXPS 2.3.19. Scans were charge referenced to the $\mathrm{C} 1 \mathrm{~s}$ aliphatic signal (component 1$)$ at 285.0 $\mathrm{eV}$ and each photoemission was fitted with the minimum number of GL (30) lineshapes (with spline-linear backgrounds) required to replicate the photoemission signals. C 1s components had FWHM constraints (1-1.3 eV), however, $\mathrm{O} 1 \mathrm{~s}$ components were unconstrained. $\mathrm{C} 1 \mathrm{~s}$ and $\mathrm{O}$ 1s 
B.E.s, FWHMs, and peak fittings were compared with existing literature reports ${ }^{29}$ to ensure samples were free from contamination and their spectra reflect that of lignin.

\section{Calculations}

\section{Pulp yields}

The pulp yields were calculated based on the mass ratios of bagasse before and after pretreatment in a dry weight basis, as described in Equation 1.

$$
\text { Pulp yield }=\frac{\text { Pretreated bagasse }_{\text {dry basis }}}{\text { Raw bagasse }_{\text {dry basis }}} \times 100 \%
$$

\section{Glucan and Hemicellulose yield in saccharification}

Yields were calculated based on the mass ratios of glucan and hemicellulose (xylan and arabinan) released on enzymatic saccharification, normalized to the initial amount used in the pretreatment (Equation 2).

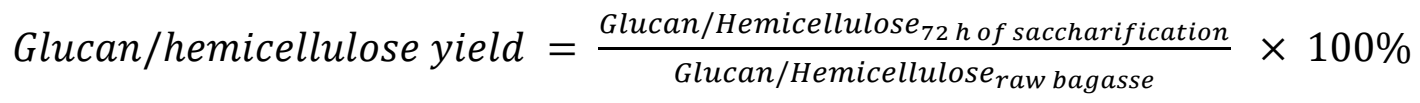

\section{Degree of delignification}


The degree of delignification was calculated based on the mass difference in the lignin content before and after pretreatment relative to the lignin content in raw bagasse in a dry weight basis, as shown in Equation 3.

$$
\text { Delignification }=\frac{\text { Lignin }_{\text {raw bagasse }} \text { Lignin }_{\text {pretreated bagasse }}}{\text { Lignin }_{\text {raw bagasse }}} \times 100 \%
$$

\section{Lignin recovery}

The lignin recovery was calculated based on the amount of lignin recovered in the precipitation step relative to the lignin content in raw bagasse in a dry weight basis (Equation 4)

$$
\text { Lignin recovery }=\frac{\text { Lignin }_{\text {recovered from the PIL }}}{\text { Lignin }_{\text {raw bagasse }}} \times 100 \%
$$

\section{Acid-base ratios (ABRs)}

Calculated ABRs were detemined by ${ }^{1} \mathrm{H}-\mathrm{NMR}$ spectroscopy by integrating the peaks for the methyl (singlet, $\delta=1.72$ ) group in the acetate anion and the more shielded methylene (doublet, $\delta=2.76$ ) group in the MEA in molecular or ionic form (Equation 5).

$$
A B R=\frac{2}{3} \times\left[\frac{\text { Area of methyl group }(\delta=1.72)}{\text { Area of methylene group }(\delta=2.76)}\right]
$$

\section{Acetamide content}


Acetamide content refers to the amount of acetamide (measured by integrating the methylene group in $\delta=3.09$ ) relative to the total solvent content (PIL/acid-base mixture). Acetamide content depends on two parameters, acetamide conversion and the ABR of the mixture. Under low ABR values, acetamide conversion was calculated based on the limiting reactant, acetic acid (Equation 6a). Conversely, under high ABR values, acetamide conversion was calculated based on the MEA (Equation 6b).

\section{Low ABRs:}

Acetamide content $=\left[\frac{\text { Area of methylene }(\delta=3.05)}{\text { Area of methylene }(\delta=3.05)+\frac{2}{3} \times \text { Area of methyl }(\delta=1.72)}\right] \times A B R$

High ABRs:

Acetamide content $=\left[\frac{\text { Area of methylene }(\delta=3.05)}{\text { Area of methylene }(\delta=3.05)+\text { Area of methylene }(\delta=2.76)}\right] \times A B R^{-1}$ 